Pacific Journal of Mathematics

ON A QUESTION OF FELT CONCERNING CHARACTER 


\title{
ON A QUESTION OF FEIT CONCERNING CHARACTER VALUES OF FINITE SOLVABLE GROUPS
}

\author{
Gideon Amit and David Chillag
}

\begin{abstract}
Let $\chi$ be an irreducible character of a finite group $G$ and let $f$ be the smallest integer such that $\{\chi(x) \mid x \in G\} \subseteq Q(\sqrt[f]{1})$. The question raised by W. Feit is: Does $G$ contain an element of order $f$. In this article we given an affirmative answer to the question for solvable groups.
\end{abstract}

Introduction. Let $G$ be a finite group and $\chi$ an irreducible complex character of $G$. Denote by $Q(\chi)$ the field obtained by adjoining the values of $\chi$ to the rational number field $Q$. For every positive integer $m$ we denote by $Q_{m}$ the field $Q(\omega)$, where $\omega$ is a primitive $m$ th root of unity. Finally, denote by $f(\chi)$ the smallest positive integer $f$ for which $Q(\chi) \subseteq$ $Q_{f}$.

The following question has been raised by Walter Feit (see e.g. [4] p. 178 ): Let $\chi$ be an irreducible complex character of a finite group $G$, does $G$ contains an element of order $f(\chi)$ ?

In this article we show that if $G$ is solvable the answer to the question is positive. Before stating this result we survey the known positive answers to the question.

Brauer ([3] Corollary 4) gave an affirmative answer in the case that $f(\chi)$ has the form $f(\chi)=p_{1}^{\alpha_{1}} p_{2}^{\alpha_{2}} \cdots p_{k}^{\alpha_{k}}$ where $\alpha_{l} \geq 2$ for all $i$ and the $p_{\imath}$ 's are primes. There is no restriction on $G$. In [5] Gow gives an affirmative answer in the case that $G$ has odd order with no restriction on $f(\chi)$. In [1], Brauer's and Gow's methods are generalized and an affirmative answer is given (Theorem 2.2 of [1]) in a case of which both Brauer's and Gow's cases are special cases. Also, it is fairly easy to prove ([2]) that if $f(\chi)$ has the form $f(\chi)=p^{\alpha} q^{\beta}, p$ and $q$ primes, the answer is also positive. The main result of this paper is:

TheOREM. Let $G$ be a finite solvable group and $\chi$ an irreducible complex character of $G$, then $G$ contains an element of order $f(\chi)$.

Most of our notation is standard and taken mainly from [6]. Some other pieces of notation will be introduced as we go along. 
2. Preliminaries and proof of the theorem. The notation $o(a)$ will be used to denote the order of the element $a$ of a group. If $G$ is a finite group and $\chi \in \operatorname{Irr}(G)$ we let $\pi(\chi)=\{p \mid p$ a prime divisor of $f(\chi)\}$. For each $p \in \pi(\chi)$ we fix a generator, $\sigma_{p}(\chi)$ of the cyclic group $\operatorname{Gal}\left(Q_{f} / Q_{f / p}\right)$, where $f=f(\chi)$. By Galois theory we have that

$$
o\left(\sigma_{p}(\chi)\right)= \begin{cases}p & \text { if } p^{2} \mid f \\ p-1 & \text { if } p^{2}+f .\end{cases}
$$

We note that if $p^{2}+f$ then $p \neq 2$. It is clear from the definitions that for all $p \in \pi(\chi)$ we have that $\chi^{\sigma_{p}(\chi)} \neq \chi$.

LEMMA 1. Let $H$ be a subgroup of the finite group $G, \chi \in \operatorname{Irr}(G)$ and $\psi \in \operatorname{Irr}(H)$.

(a) If $Q(\chi) \subseteq Q(\psi)$ then $f(\chi) \mid f(\psi)$.

(b) If $Q(\psi) \subseteq Q(\chi)$ and $\psi^{\sigma_{p}(\chi)} \neq \psi$ for all $p \in \pi(\chi)$, then $f(\chi)=$ $f(\psi)$.

Proof. If $Q(\chi) \subseteq Q(\psi)$ then $Q_{f(\chi)} \subseteq Q_{f(\psi)}$ and (a) follows. As $\psi^{\sigma_{p}(\chi)}$ $\neq \psi$ is equivalent to $Q(\psi) \nsubseteq Q_{f(x) / p}$ we get that (b) holds.

Proposition 2. Let $\chi \in \operatorname{Irr}(G), f=f(\chi)$ and $\pi=\pi(\chi)$. If $G$ contains no element of order $f(\chi)$ then there exist $p \in \pi$ such that:

(a) $p^{2}+f$, and

(b) $\chi^{\sigma_{p}(\chi)}=\chi^{\tau}$ for some $\tau \in \operatorname{Gal}\left(Q_{f} / Q_{p}\right)$.

Proof. Let $\sigma_{q}=\sigma_{q}(\chi)$ for each $q \in \pi$ and set $\mathscr{G}=\operatorname{Gal}\left(Q_{f} / Q\right)$. Denote by $A$ the abelian subgroup of the ring of class functions of $G$ that is generated by $\left\{\chi^{\sigma} \mid \sigma \in \mathscr{G}\right\}$. For each $\sigma \in \mathscr{G}$ and $\alpha \in A$ define $\alpha \cdot \sigma=\alpha^{\sigma}$. Then $A$ becomes a $Z \mathscr{G}$-module, where $Z$ is the ring of integers.

Let $g \in G$. Then $o(g)$ is not divisible by the full $q$-part of $f$ for some $q \in \pi$. Then $\alpha(g)^{\sigma_{q}}=\alpha(g)$ for all $\alpha \in A$. It follows that if $\beta \in A$. $\left(\sigma_{q}-1\right)$ then $\beta(g)=0$. Since each $g \in G$ has such a $q \in \pi$, we get that if $\beta \in A \cdot \prod_{q \in \pi}\left(\sigma_{q}-1\right)$, then $\beta(g)=0$ for all $g \in G$. This shows that $\prod_{q \in \pi}\left(\sigma_{q}-1\right)$ annihilates $A$ and in particular it annihilates $\chi$.

Let $\pi_{0}$ be a subset of $\pi$ minimal such that $\chi \cdot \Pi_{q \in \pi_{0}}\left(\sigma_{q}-1\right)=0$. Let $p$ be the largest prime in $\pi_{0}$ and set $\pi_{1}=\pi_{0}-\{p\}$. Write $\varepsilon=$ $\Pi_{q \in \pi_{1}}\left(\sigma_{q}-1\right)$, then $\chi \cdot\left(\sigma_{p}-1\right) \varepsilon=0$ and the minimality of $\pi_{0}$ implies that $\chi \cdot \varepsilon \neq 0$. Hence $\chi \cdot \sigma_{p} \varepsilon=\chi \cdot \varepsilon \neq 0$. An irreducible constituent of $\chi \cdot \sigma_{p} \varepsilon=\chi^{\sigma_{p}} \cdot \varepsilon$ has a form $\chi \cdot \sigma_{p} \mu=\chi \cdot \nu$ where $\mu, \nu$ are in the abelian group $B=\left\langle\sigma_{q} \mid q \in \pi_{1}\right\rangle$. Thus $\chi \cdot \sigma_{p}=\chi \cdot \nu \mu^{-1}$. Let $\tau=\nu \mu^{-1}$, then 
$\chi^{\sigma_{p}}=\chi^{\tau}$ and $\tau \in B$. We note that if $q \in \pi_{1}$, then $\sigma_{q} \in \operatorname{Gal}\left(Q_{f} / Q_{f / q}\right) \subseteq$ $\operatorname{Gal}\left(Q_{f} / Q_{p}\right)$ as $q \neq p$. It follows that $\tau \in B \subseteq \operatorname{Gal}\left(Q_{f} / Q_{p}\right)$ as required.

Finally, we claim that $p^{2}+f$. For if $p^{2} \mid f$, then $o\left(\sigma_{p}\right)=p$ and the equality $\chi^{\sigma_{p}}=\chi^{\tau}$ implies that $p \mid o(\tau)$. On the other hand the maximality of $p$ implies that $o\left(\sigma_{q}\right) \leq q<p$ for all $\sigma_{q} \in B$. As $\tau$ is a product of elements of $B$ we get that $p+o(\tau)$, a contradiction.

Definitions. (1) Let $G$ be a finite solvable group. A $p$-chief factor of $G, K / L$, is called distinguished if $p+|G: K|$. There is in this case a unique conjugacy class of complements of $K / L$ in $G$, a complement being a subgroup $H$ of $G$ such that $G=K H$ and $K \cap H=L,|H|<|G|$.

(2) If $N \triangleleft G$ and $\theta \in \operatorname{Irr}(N)$ we define $\operatorname{Irr}(G \mid \theta)=\left\{\chi \in \operatorname{Irr}(G) \mid\left[\chi_{N}, \theta\right]\right.$ $\neq 0\}$.

The next lemma sums up some known facts from character correspondence theory that will be needed in the proof of the Theorem.

LEMMA 3. Let $K / L$ be a distinguished chief factor of the solvable finite group $G$ and let $H$ be a complement of $K / L$. Suppose that $\chi \in \operatorname{Irr}(G)$ is primitive. Then $\chi_{K}$ and $\chi_{L}$ have, each, a unique irreducible constituent, $\theta$ and $\phi$ respectively, and there are just two possiblities:

(i) $\theta_{L}=\phi$. In this case the mapping $\mu \rightarrow \mu_{H}$ is a bijection from $\operatorname{Irr}(G \mid \theta)$ to $\operatorname{Irr}(H \mid \phi)$. In particular: $\chi_{H}=\xi \in \operatorname{Irr}(H \mid \phi)$ and $\xi$ and $\theta$ together uniquely determine $\chi$. Thus if $\sigma \in \mathrm{Gal}\left(Q_{f(x)} / Q\right)$ and $\xi^{\sigma}=\xi$ and $\theta^{\sigma}=\theta$, then $\chi^{\sigma}=\chi$.

(ii) $\theta_{L}=e \phi$ with $e^{2}=|K: L|$. In this case there is a canonically defined bijection $\operatorname{Irr}(G \mid \theta) \rightarrow \operatorname{Irr}(H \mid \phi)$. If $\chi \rightarrow \xi$ in this bijection then each of $\chi$ and $\xi$ uniquely determines $\theta$ and $\phi$ and so each determines the other. It follows by Galois theory that $Q(\chi)=Q(\xi)$.

Proof. See [6], [7] and [8].

Proposition 4. Let $G$ be a finite solvable group and $\chi \in \operatorname{Irr}(G)$. Assume that there exists no proper subgroup $X$ of $G$ and $\psi \in \operatorname{Irr}(X)$ such that $f(\chi)$ divides $f(\psi)$. For a $p \in \pi=\pi(\chi)$, let $K / L$ be a distinguished $p$-chief factor and let $H$ be a complement of $K / L$. If $\sigma_{p}=\sigma_{p}(\chi)$, then

(a) $\chi_{H}=\xi \in \operatorname{Irr}(H)$ and $\xi^{\sigma_{p}}=\xi$.

(b) $\chi_{K}=a \theta, \theta \in \operatorname{Irr}(K)$, a a positive integer and $\theta^{\sigma_{p}} \neq \theta$.

(c) $\theta_{L}=\phi \in \operatorname{Irr}(L)$ and $\phi_{p}^{\sigma_{p}}=\phi$.

Proof. Assume that the Proposition is false and choose $p$ as large as possible to get a counterexample. Then the conclusions of the Proposition 
are false for some distinguished $p$-chief factor, $K / L$, and they hold for distinguished $q$-chief factors for $q>p, q \in \pi$.

If $\chi$ is induced from some proper subgroup $X$ of $G$, say $\chi=\psi^{G}$, $\psi \in \operatorname{Irr}(X)$, then $Q(\chi) \subseteq Q(\psi)$ and so $f(\chi)$ divides $f(\psi)$. This is a contradiction. Thus $\chi$ is primitive so we apply Lemma 3 . Hence $\chi_{K}=a \theta$ for some $\theta \in \operatorname{Irr}(K)$ and a natural number $a$. Let $\phi$ be the unique irreducible constituent of $\chi_{L}$. If $\theta_{L}=e \phi$ with $e^{2}=|K: L|$ then there is a $\xi \in \operatorname{Irr}(H)$ with $Q(\chi)=Q(\xi)$. Therefore $f(\chi)=f(\xi)$, a contradiction. Thus $\theta_{L}=\phi \in \operatorname{Irr}(L)$ and $\chi_{H}=\xi \in \operatorname{Irr}(H)$.

Now $Q(\xi) \subseteq Q(\chi)$ but $f(\xi) \neq f(\chi)$ and thus there exist $q \in \pi$ with $\xi^{\sigma_{q}}=\xi$ (see Lemma 1). Note that $\xi_{L}=\chi_{L}=a \phi$ and hence $\phi^{\sigma_{q}}=\phi$. Since $\chi^{\sigma_{q}} \neq \chi$ and $\chi$ is uniquely determined by $\xi$ and $\theta$, we must have $\theta^{\sigma_{q}} \neq \theta$. If $q=p$, then $K / L$ is not a counterexample contrary to hypothesis. Therefore $q \neq p$.

$\operatorname{Next}\left(\theta^{\sigma_{q}}\right)_{L}=\phi^{\sigma_{q}}=\phi$ and so $\theta$ and $\theta^{\sigma_{q}}$ are two distinct extensions of $\phi$ and hence $\theta$ and $\theta^{\sigma_{q}}$ are two distinct irreducible constituents of $\phi^{K}$. By [6] Corollary 6.17 we get that $\theta^{\sigma_{q}}=\lambda \theta$ for some $\lambda \in \operatorname{Irr}(K / L), \lambda \neq 1$. Since $o(\lambda)=p \neq q$ we have that $\lambda^{\sigma_{q}}=\lambda$. Thus for every positive integer $k$ we have $\boldsymbol{\theta}^{\left(\sigma_{q}\right)^{k}}=\boldsymbol{\theta} \lambda^{k}$. By taking $k=o\left(\sigma_{q}\right)$ we obtain that $\boldsymbol{\theta}=\boldsymbol{\theta} \lambda^{k}$ and hence $\lambda^{k}=1$ by (6.17) of [6]. It follows that $p \mid o\left(\sigma_{q}\right)$. Recall that $o\left(\sigma_{q}\right)=q$ or $q-1$ and $p \neq q$. Therefore $p \mid q-1$ and $q>p$.

Hence, if $K_{0} / L_{0}$ is any distinguished $q$-chief factor, then the conclusions of the Proposition hold. This means that $\chi_{K_{0}}=a_{0} \theta_{0},\left(\theta_{0}\right)_{L_{0}}=\phi_{0}$, $\theta_{0}^{\sigma_{q}} \neq \theta_{0}, \phi_{0}^{\sigma_{q}}=\phi_{0}$ where $\theta_{0} \in \operatorname{Irr}\left(K_{0}\right), \phi_{0} \in \operatorname{Irr}\left(L_{0}\right)$ and $a_{0}$ is a positive integer. If $q \| G: K \mid$, then we can choose $K_{0} / L_{0}$ with $K \subseteq L_{0}$. Then $\chi_{L_{0}}=a_{0} \phi_{0}$ and so $a \theta=\chi_{K}=a_{0}\left(\phi_{0}\right)_{K}$ and hence $\left(\phi_{0}\right)_{K}=\left(a / a_{0}\right) \theta$. Since $\phi_{0}^{\sigma_{q}}=\phi_{0}$ we get that $\theta^{\sigma_{q}}=\theta$, a contradiction. Therefore $q+|G: K|$. Now we choose $K_{0} / L_{0}$ with $K_{0} \subseteq L$ and as above we get that $\phi_{K_{0}}$ is a multiple of $\theta_{0}$. But $\phi^{\sigma_{q}}=\phi$ and this yields $\left(\theta_{0}\right)^{\sigma_{q}}=\theta_{0}$, a contradiction. This completes the proof.

Proof of the Theorem. Let $G$ be a minimal counterexample. If $G$ contains a proper subgroup $H$ with $\psi \in \operatorname{Irr}(H)$ such that $f(\chi) \mid f(\psi)$, then by induction $H$ contains an element $h$ with $o(h)=f(\psi)$. Then there exist $g \in\langle h\rangle$ with $o(g)=f(\chi)$, a contradiction. Hence $G$ satisfies the assumptions of Proposition 4 and therefore its conclusions. Set $f=f(\chi), \pi=$ $\pi(\chi)$ and $\sigma_{q}=\sigma_{q}(\chi)$ for all $q \in \pi$. By Proposition 2 we can choose $p \in \pi$ with $p^{2}+f$ and $\tau \in \operatorname{Gal}\left(Q_{f} / Q_{p}\right)$ such that $\chi^{\sigma_{p}}=\chi^{\tau}$. Clearly $p \neq 2$. Let $K / L$ be a distinguished $p$-chief factor and $H$ a complement of $K / L$. Then by Proposition 4 we get: $\chi_{H}=\xi, \chi_{K}=a \theta, \theta_{L}=\phi$ where $\xi \in$ 
$\operatorname{Irr}(H), \theta \in \operatorname{Irr}(K), \phi \in \operatorname{Irr}(L)$ and $a$ a positive integer. Moreover $\xi^{\sigma_{p}}=\xi$, $\phi^{\sigma_{p}}=\phi$ but $\theta_{p}^{\sigma_{p}} \neq \theta$.

Since $\phi^{\sigma_{p}}=\phi$, we have that $Q(\phi) \subseteq Q_{f / p}$ and as $p^{2}+f$ we conclude that $\phi$ is $p$-rational. As $p \neq 2$, Theorem (6.30) of [6] implies that $\phi$ has a unique $p$-rational extension $\mu \in \operatorname{Irr}(K)$. As $\theta_{L}=\mu_{L}=\phi$ we get by (6.17) of [6] that $\theta=\lambda \mu$ for some $\lambda \in \operatorname{Irr}(K / L)$. Note that $\phi$ and $\mu$ uniquely determine each other so that $Q(\mu)=Q(\phi) \subseteq Q(\theta) \subseteq Q(\chi) \subseteq Q_{f}$. Also $\mu^{\sigma_{p}}$ is a $p$-rational extension of $\phi^{\sigma_{p}}=\phi$ and by the uniqueness we have $\mu^{\sigma_{p}}=\mu$.

Note that $\lambda(g) \in Q_{p}$ for all $g \in K / L$ and so $\lambda^{\tau}=\lambda$. Also, $\sigma_{p}$ agrees with $\tau$ on $Q(\chi)$. Since $Q(\theta) \subseteq Q(\chi)$, this yields

$$
\theta^{\sigma_{p}}=\theta^{\tau}=(\lambda \mu)^{\tau}=\lambda^{\tau} \mu^{\tau}=\lambda \mu^{\sigma_{p}}=\lambda \mu=\theta .
$$

This contradiction completes the proof.

Acknowledgment. We wish to thank Professor I. Martin Isaacs for carefully reading our manuscript and for providing us detailed suggestions which simplified the proof of the theorem considerably.

\section{REFERENCES}

[1] G. Amit and D. Chillag, Character Values, Conjugacy Classes and a Problem of Feit, to appear in Houston J. of Math.

[2] _ On a question of W. Feit concerning values of characters of finite groups. Proceedings of the fourth international conference on representations of algebras. Edited by V. Dlab. Carleton-Ottawa Mathematical Lecture Notes Series.

[3] R. Brauer, A note on theorems of Burnside and Blichfeldt, Proc. Amer. Math. Soc., 15 (1964), 31-34.

[4] W. Feit, Some consequences of the classification of finite simple groups, the Santa-Cruz conference on finite groups, Proc. of Symposia in pure math. vol. 37, Amer. Math. Soc., (1980), 175-181.

[5] R. Gow, Character values of groups of odd order and a question of Feit, J. Algebra, 68 (1981), 75-78.

[6] I. M. Isaacs, Character Theory of Finite Groups, Academic Press, New York, 1976.

[7] __, Characters of solvable and symplectic groups, Amer. J. Math., 85 (1973), 594-635.

[8] T. R. Wolf, Character correspondences in solvable groups, Illinois J. Math., 22 (1978), $327-340$.

Received December 7, 1984 and in revised form July 9, 1985.

TECHNION-ISRAEL INSTITUTE OF TECHNOLOGY HAIFA 32000, ISRAEL 



\section{PACIFIC JOURNAL OF MATHEMATICS EDITORS}

\author{
V. S. VARAdarajan (Managing Editor) \\ University of California \\ Los Angeles, CA 90024 \\ Hebert Clemens \\ University of Utah \\ Salt Lake City, UT 84112 \\ Charles R. DePrima \\ California Institute of Technology \\ Pasadena, CA 91125
}

R. FINN

Stanford University

Stanford, CA 94305

HeRManN FLASChKa

University of Arizona

Tucson, AZ 85721

RAMESH A. GANGOlli

University of Washington

Seattle, WA 98195

ROBION KIRBY

University of California

Berkeley, CA 94720

\author{
C. C. MOORE \\ University of California \\ Berkeley, CA 94720 \\ H. SAMELSON \\ Stanford University \\ Stanford, CA 94305 \\ HAROLD STARK \\ University of California, San Diego \\ La Jolla, CA 92093
}

\section{ASSOCIATE EDITORS}

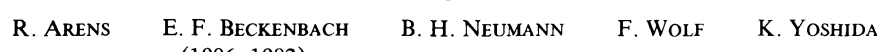

(1906-1982)

\section{SUPPORTING INSTITUTIONS}

\begin{abstract}
UNIVERSITY OF ARIZONA
UNIVERSITY OF BRITISH COLUMBIA

CALIFORNIA INSTITUTE OF TECHNOLOGY

UNIVERSITY OF CALIFORNIA

MONTANA STATE UNIVERSITY

UNIVERSITY OF NEVADA, RENO

NEW MEXICO STATE UNIVERSITY

OREGON STATE UNIVERSITY
\end{abstract}

\author{
UNIVERSITY OF OREGON \\ UNIVERSITY OF SOUTHERN CALIFORNIA \\ STANFORD UNIVERSITY \\ UNIVERSITY OF HAWAII \\ UNIVERSITY OF TOKYO \\ UNIVERSITY OF UTAH \\ WASHINGTON STATE UNIVERSITY \\ UNIVERSITY OF WASHINGTON
}

The Supporting Institutions listed above contribute to the cost of publication of this Journal, but they are not owners or publishers and have no responsibility for its content or policies.

Mathematical papers intended for publication in the Pacific Journal of Mathematics should be in typed form or offset-reproduced (not dittoed), double spaced with large margins. Please do not use built up fractions in the text of the manuscript. However, you may use them in the displayed equations. Underline Greek letters in red, German in green, and script in blue. The first paragraph must be capable of being used separately as a synopsis of the entire paper. In particular it should contain no bibliographic references. Please propose a heading for the odd numbered pages of less than 35 characters. Manuscripts, in triplicate, may be sent to any one of the editors. Please classify according to the scheme of Math. Reviews, Index to Vol. 39. Supply name and address of author to whom proofs should be sent. All other communications should be addressed to the managing editor, or Elaine Barth, University of California, Los Angeles, California 90024.

There are page-charges associated with articles appearing in the Pacific Journal of Mathematics. These charges are expected to be paid by the author's University, Government Agency or Company. If the author or authors do not have access to such Institutional support these charges are waived. Single authors will receive 50 free reprints; joint authors will receive a total of 100 free reprints. Additional copies may be obtained at cost in multiples of 50 .

The Pacific Journal of Mathematics is issued monthly as of January 1966. Regular subscription rate: $\$ 190.00$ a year (5 Vols., 10 issues). Special rate: $\$ 95.00$ a year to individual members of supporting institutions.

Subscriptions, orders for numbers issued in the last three calendar years, and changes of address should be sent to Pacific Journal of Mathematics, P.O. Box 969, Carmel Valley, CA 93924, U.S.A. Old back numbers obtainable from Kraus Periodicals Co., Route 100, Millwood, NY 10546.

The Pacific Journal of Mathematics at P.O. Box 969, Carmel Valley, CA 93924 (ISSN 0030-8730) publishes 5 volumes per year. Application to mail at Second-class postage rates is pending at Carmel Valley, California, and additional mailing offices. Postmaster: Send address changes to Pacific Journal of Mathematics, P.O. Box 969, Carmel Valley, CA 93924.

PUBLISHED BY PACIFIC JOURNAL OF MATHEMATICS, A NON-PROFIT CORPORATION

Copyright $\odot 1986$ by Pacific Journal of Mathematics 


\section{Pacific Journal of Mathematics}

\section{Vol. 122, No. 2 \\ February, 1986}

Gideon Amit and David Chillag, On a question of Feit concerning character values of finite solvable groups ......................257

Constantin Gelu Apostol and Frank Larkin Gilfeather, Isomorphisms modulo the compact operators of nest algebras ................263

Parviz Azimi and James Neil Hagler, Examples of hereditarily $l^{1}$ Banach

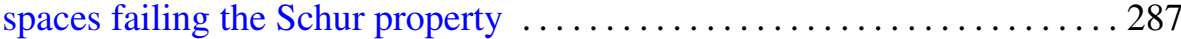

Brian Evan Blank, Boundary behavior of limits of discrete series representations of real rank one semisimple groups . . . . . . . . . . 299

Jeffrey Carroll, Some undecidability results for lattices in recursion theory

Gerald Howard Cliff and Alfred Rheinhold Weiss, Crossed product and

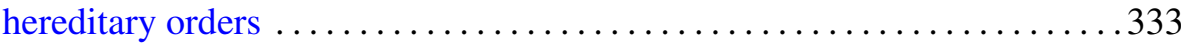

Ralph Cohen, Realizing transfer maps for ramified coverings . . . . . . . . 347

Ronald James Evans, Hermite character sums . .................. 357

C. L. Frenzen and Roderick Sue-Chuen Wong, Asymptotic expansions of the Lebesgue constants for Jacobi series . . . . . . . . . . . . . . . . 391

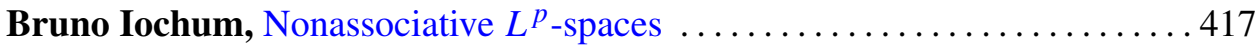

John McDonald, Unimodular approximation in function algebras ....... 435

John Robert Quine, Jr., Ramification and unintegrated value distribution . . 441

Marc Raphael, Commutants of quasisimilar subnormal operators ........ 449

Parameswaran Sankaran and Peter Zvengrowski, On stable

parallelizability of flag manifolds

Helga Schirmer, A relative Nielsen number

Barry Simon, Schrödinger semigroups on the scale of Sobolev spaces . . . . . 475

Viakalathur Shankar Sunder, Stochastic integration in Fock space

Jan de Vries, A note on the $G$-space version of Glicksberg's theorem 